\title{
Investigation of lncRNA H19 in prostate cancer cells and secreted exosomes upon androgen stimulation or androgen receptor blockage
}

\author{
Ozgur E, Gezer U \\ Department of Basic Oncology, Oncology Institute, Istanbul University, Istanbul, Turkey. \\ ugurd@istanbul.edu.tr
}

\begin{abstract}
OBJECTIVES: In the present study, cellular or exosomal expression of $\mathrm{H} 19$, an oncofetal IncRNA gene, was evaluated during androgen stimulation via dihydrotestosterone (DHT) or AR blockage via enzalutamide in cultured hormone-sensitive Pca cells which overexpres AR (LNCaP-AR ${ }^{+}$.

BACKGROUND: Prostate cancer ( $\mathrm{PCa}$ ) is an androgen-dependent disease. Androgen receptor (AR) antagonists (i.e. enzalutamide) have been used for the treatment of patients with metastatic castrationresistant prostate cancer (CRPC). Exosomes and their contents (non-coding RNA) play an important role in tumor development and progression.

METHODS: Cells were treated with DHT (10 nM) and/or enzalutamide (10 uM) for $24 \mathrm{~h}$. Cellular and exosomal expression of $\mathrm{H} 19$ was investigated using a quantitative polymerase chain reaction assay. RESULTS: Our findings reveal that cellular $\mathrm{H} 19$ expression decreased approximately 2.3 fold in mean upon androgen stimulation of Pca cells. AR blockage using enzalutamide restored DHT effect and we found increased $H 19$ expression $(\leq 2.5$-fold, $p<0.05)$ upon the combined use of DHT and enzalutamide compared to control cells. Similar to its cellular effect, DHT treatment also led to declined exosomal expression of $H 19$ ( $\leq 3$-fold, $p<0.0001$ ). Restorative effect of enzalutamide on decreased H19 expression induced by androgen stimulation was not observed in exosomes.

CONCLUSION: This experimental study provides evidence that $\mathrm{H} 19$ might be involved in androgen receptor pathway. Further research is needed to explore the role of $\mathrm{H} 19$ in Pca and intercellular communication via exosomes (Fig. 2, Ref. 32). Text in PDF www.elis.sk.

KEY WORDS: prostate cancer, exosomes, H19, androgen, enzalutamide.
\end{abstract}

\section{Introduction}

Prostate cancer (PCa), a malignant disease of the prostate gland, is the second most common cancer in men worldwide (1). $\mathrm{PCa}$ is associated with androgen receptor (AR) pathway as its development is androgen-dependent. Thus, the suppression of androgen pathway by surgical or medical castration is the approach of the treatment of this disease. Despite a high response rate with androgen deprivation, as consequence of continuous AR activation, many patients with metastatic disease progress to castrationresistant prostate cancer (CRPC) (2). In recent years, the second generation AR inhibitor enzalutamide (3) and androgen biosynthesis inhibitor abiraterone acetate (4) have been developed as

Department of Basic Oncology, Oncology Institute, Istanbul University, Istanbul, Turkey

Address for correspondence: E. Ozgur, $\mathrm{PhD}$, Istanbul University, Oncology Institute, Department of Basic Oncology, Millet Cad., 34093, CapaFatih, Istanbul, Turkey.

Phone: +90.2124142434 , Fax: +90.2125348078

Acknowledgement: This work was supported by the Istanbul University Scientific Projects Coordination Unit (Project No: 27356). treatment options in patients with metastatic CRPC. Enzalutamide acts in multiple ways; it blocks the binding of androgens to the $\mathrm{AR}$ and the transition of active AR into the nucleus and prevents the binding of the DNA to the AR (5).

Prostate specific antigen (PSA) is the most widely used biomarker in the management of PCa. Serum levels of PSA also increase in other prostate disorders such as prostatitis or benign prostatic hyperplasia (BPH), its diagnostic value is limited (6). Therefore, identification of more specific biomarkers will improve diagnostic accuracy of PCa.

Recently, exosomes which are membrane-bound secreted vesicles $(30-150 \mathrm{~nm})$, constitute a valuable source for biological marker discovery (7-11). Exosomes are involved in the regulation of many physiological or pathological processes including tumor development and progression. It has been shown that exosomes contain most types of macromolecules including lipids, proteins, DNA, messenger RNA microRNA and long noncoding RNAs (lncRNAs). They mediate intercellular communication by delivering their loads to target cells. Recent studies reveal increased levels of many non-coding RNAs in exosomes extracted from blood plasma in many cancer types, including PCa (12-15). We have previously demonstrated that some lncRNAs were enriched 
in exosomes released from cultured cells relative to their cellular levels (16).

LncRNAs are longer than 200 nucleotides with restricted protein-encoding ability $(17,18)$. Aberrant expression of many lncRNAs were detected in multiple human cancers, such as colorectal, lung, breast, liver, bladder cancer, and PCa $(19,22)$. LncRNAs play critical roles in all stages of PCa development and progression (23). H19, an oncofetal RNA, is the first discovered lncRNA molecule and has been shown to possess regulatory functions in cancer development (24). In this experimental study, we aimed to study whether cellular or exosomal levels of $H 19$ are adjusted during androgen stimulation or AR inhibition in cultured PCa cells using a cell line overexpressing AR as the cellular model.

\section{Materials and methods}

\section{Cell culture}

We used LNCaP-AR+ cells, kindly gifted by Charles Sawyers Lab (Memorial Sloan Kettering Cancer Center, New York, NY, USA) which are androgen sensitive and modified to overexpress AR. Cells were cultured in the RPMI-1640 medium containing NaHCO3 (3.7 g/l), glucose (1 g/l) and stable glutamine (Biochrom, Berlin, Germany) supplemented with $10 \%$ fetal calf serum (FCS) (Biochrom) and antibiotics under standard conditions $\left(37^{\circ} \mathrm{C}\right.$ and $5 \% \mathrm{CO}_{2}$ humidity).

\section{Androgen stimulation and androgen receptor blockade}

Cells were seeded at a density of $2 \times 10^{5}$ cells in culture plates and grown for 72 hours in the growth medium supplemented with exosome-depleted charcoal/dextran stripped FCS. The medium was then replaced by a fresh one containing dihydrotestosterone (DHT) (Sigma-Aldrich, USA), enzalutamide (kindly donated by Astellas Inc., Northbrook, IL, USA) or the control (dimethyl sulfoxide, DMSO). DHT is active metabolite of androgen. Cells were further grown $24 \mathrm{~h}$ and harvested along with culture medium and stored for subsequent analysis.

Isolation of exosomes from culture medium and of exosomal RNA

Exosome extraction from the culture medium was performed using the Total Exosome Isolation reagent (Thermo Fisher Scientific, Inc., Waltham, MA, USA) according to the manufacturer's instructions. RNA was isolated from the extracted exosomes using the Total Exosome RNA \& Protein Isolation kit (Thermo Fisher Scientific, Inc.) as per the manufacturer's instructions. Total cellular RNA was extracted using the RNA isolation solution (Roche Diagnostics $\mathrm{GmbH}$ ) according to the manufacturer's instructions.

\section{Quantitative PCR}

Total RNA and exosomal RNA were used for complementary DNA (cDNA) synthesis using a First-Strand cDNA Synthesis kit (Thermo Scientific, Waltham, MA, USA), in accordance with the manufacturers' instructions. Expression analysis was performed using the LightCycler 480 instrument (Roche) and SYBR Green (Roche) as the fluorescent molecule. H19 expression levels were normalized with the internal control 18S rRNA. The PCR program included an initial hot start of $10 \mathrm{~min}$, followed by 45 cycles of amplification. Each cycle consisted of a denaturation step at $95^{\circ} \mathrm{C}$ for $10 \mathrm{~s}$, annealing starting at $60^{\circ} \mathrm{C}$ for $20 \mathrm{~s}$ and decreasing $2{ }^{\circ} \mathrm{C}$ every 2 cycles until $55{ }^{\circ} \mathrm{C}$, as well as amplification at $72{ }^{\circ} \mathrm{C}$ for $30 \mathrm{~s}$. The results of three different experiments were used for the calculation of expression level of H19.

\section{Statistical analysis}

We assessed the results of three independent cell culture experiments. Changes in gene expression levels relative to control cells were expressed as 'fold changes' and mean values were statistically compared using the ANOVA Tukey multiple comparison analysis method. $\mathrm{P}<0.05$ was considered as a level of significance. Statistical analyses were conducted using the GraphPad Prism 5 software.

\section{Results}

We studied the effect of androgen stimulation of $\mathrm{LNCaP}^{-\mathrm{AR}^{+}}$ cells and AR suppression via enzalutamide on cellular and exosomal expression of H19. DHT stimulation $(10 \mathrm{nM})$ of $\mathrm{LNCaP}_{-} \mathrm{AR}^{+}$ cells for $24 \mathrm{~h}$ resulted in a 2.3 -fold decrease of cellular expression of H19 compared with control cells. The extent of the decline of H19 level was not statistically significant. Treatment of cells with enzalutamide (10 uM) alone had no effect on the H19 expression. Interestingly, upon the combined use of DHT and enzalutamide on $\mathrm{LNCaP}-\mathrm{AR}^{+}$cells we noticed restoration of the DHT effect by enzalutamide as H19 expression in cells treated with DHT and enzalutamide was 2.5 -fold higher than that in control cells and 5- fold higher than in cells stimulated with DHT $(\mathrm{p}<0.05)$ (Fig. 1).

Next, we examined the expression of $\mathrm{H} 19$ in exosomes extracted from culture media secreted by $\mathrm{LNCaP}-\mathrm{AR}^{+}$cells. Interestingly, we noticed that $\mathrm{H} 19$ was highly enriched in exosomes compared with its cellular level. Similar to its cellular expression, exosomal level of H19 was approx. 3-fold declined in DHT-treated cells

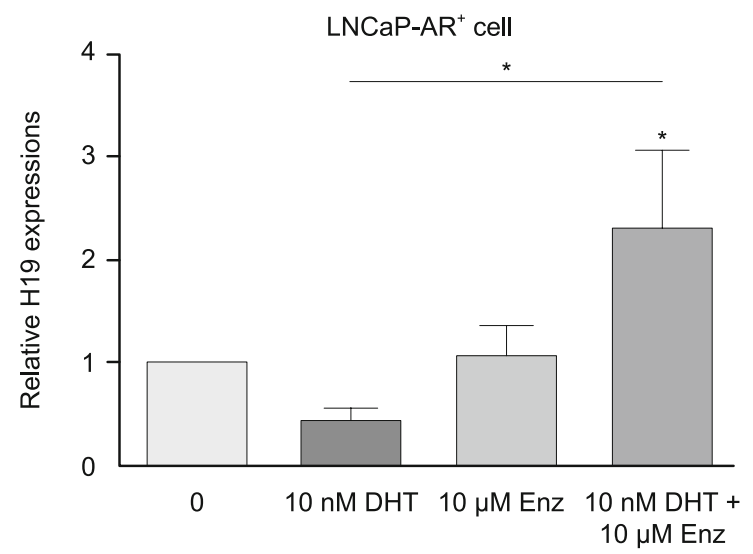

Fig. 1. Expression of IncRNA H19 in DHT or enzalutamide treated LNCaP-AR ${ }^{+}$cells. Results of three independent cell culture experiments were used to calculate the average value of relative gene expression. Each column represents mean $+\mathrm{SE}$ and ${ }^{*} \mathrm{p}<0.05$ indicates statistically significant differences compared to untreated cells and DHT treated cells. 


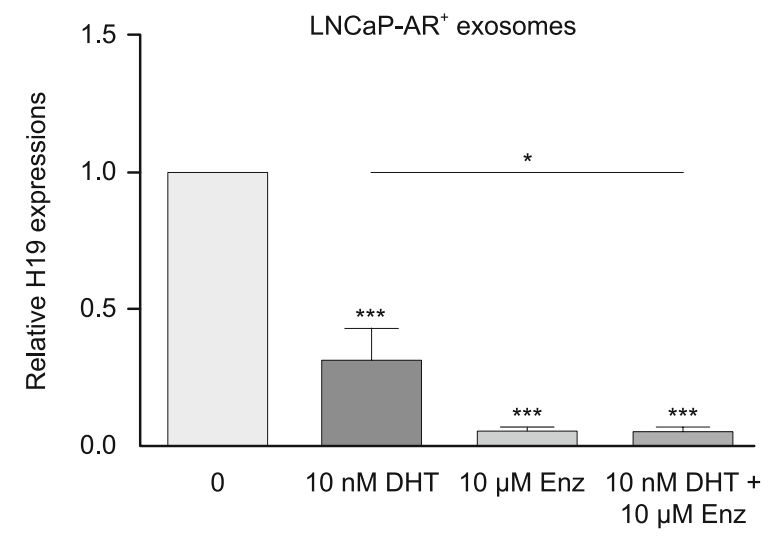

Fig. 2. Exosomal expression of IncRNA H19 in DHT or enzalutamide

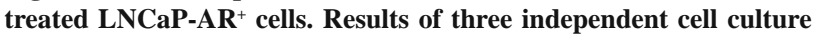
experiments were used to calculate the average value of relative gene expression. Each column represents mean $+\mathrm{SE}$ and ${ }^{* * *} \mathbf{p}<0.0001$ indicates statistically significant differences compared to untreated cells. ${ }^{*} \mathbf{p}<0.05$ indicates significant change compared with DHT treated cells.

$(\mathrm{p}<0.0001)$. Intriguingly, the effect of enzalutamide was more pronounced on exosomal expression of $\mathrm{H} 19$ as we detected approximately 19-fold decrease of H19 level in enzalutamide-exposed cells $(\mathrm{p}<0.0001)$. The combination of DHT and enzalutamide resulted in a more reduced expression of H19 in exosomes (approximately 22-fold, p < 0.0001) (Fig. 2).

\section{Discussion}

In this study, we aimed to investigate the effect of androgen pathway on the exosomal expression of $\mathrm{H} 19$ gene in AR-overexpressing prostate cancer cells $\left(\mathrm{LNCaP}-\mathrm{AR}^{+}\right)$. The $\mathrm{H} 19$ is a typical molecule for lncRNA genes that are maternally expressed and paternally suppressed. It has been reported to exert oncogenic functions in multiple cancers whereas some studies have also described a tumor suppressor role, depending on the type of cancer and cellular content $(25,26)$. H19 negatively regulates the p53 protein and cell cycle progression (26) or acts as a molecular sponge to regulate the let-7 family of microRNAs (miRNAs) (27). However, in a recent study, H19 and H19-produced miR-675 were both significantly downregulated in metastatic prostate cancer cells compared to non-metastatic prostate cancer cells (28). These studies lead to contradictory findings on whether H19 RNA is an oncogenic or tumor suppressor.

We revealed that hormone treatment of PCa cells leads to decreased expression of cellular H19. Similar finding has been reported by Berteaux et al (29). We also found that blocking the androgen receptor by enzalutamide restores the testosterone effect suggesting that H19 might have tumor suppressor activity in prostate cancer.

We showed that $\mathrm{H} 19$ was enriched in secreted exosomes. This is consistent with our previous report including some other lncRNAs that have been found to accumulate in secreted exosomes (16). This may be associated with any role of H19 in cellular commu- nication as reported in liver cancer cells in which exosomal H19 has been shown to be transferred to endothelial cells and regulate various processes via VEGF (30). Decreased expression of H19 in $\mathrm{LNCaP}_{-} \mathrm{AR}^{+}$upon DHT treatment was also reflected by exosomal level of H19. Even if enzalutamide treatment alone had no significant effect on cellular expression of H19, exosomal level of H19 was decline upon enzalutamide. This effect was increased with combined use of DHT and enzalutamide. The basis of this observation is clear at the moment but might be associated with the role of H19 in intercellular communication $(31,32)$.

In conclusion, our experimental study provides evidence that H19 might be involved in androgen/receptor pathway as testosterone treatment of hormone- dependent receptor-overexpressing cells suppresses H19. Accordingly, exosomal levels of H19 also decline in parallel to its cellular levels. Blockage of AR by enzalutamide restores the testosterone effect on $\mathrm{H} 19$ expression in cells but not in exosomes. Further research is needed to explore the role of H19 in Pca and intercellular communication via exosomes.

\section{References}

1. Siegel RL, Miller KD, Jemal A. Cancer statistics, 2018. CA Cancer J Clin 2018; 68 (1): 7-30.

2. Filella X, Foj L. Prostate Cancer Detection and Prognosis: From Prostate Specific Antigen (PSA) to Exosomal Biomarkers. Int J Mol Sci 2016; 17 (11).

3. Beer TM, Armstrong AJ, Rathkopf DE et al. PREVAIL Investigators. Enzalutamide in metastatic prostate cancer before chemotherapy. N Engl J Med 2014; 371 (5): 424-433.

4. de Bono JS, Logothetis CJ, Molina A et al. COU-AA-301 Investigators. Abiraterone and increased survival in metastatic prostate cancer. $\mathrm{N}$ Engl J Med 2011; 364 (21): 1995-2005.

5. Tran C, Ouk S, Clegg NJ et al. Development of a second-generation antiandrogen for treatment of advanced prostate cancer. Science 2009; 324 (5928): 787-790.

6. Hoffman RM. Clinical practice. Screening for prostate cancer. N Engl J Med; 365 (21): 2013-2019.

7. Khan S, Jutzy JM, Aspe JR, McGregor DW, Neidigh JW, Wall NR. Survivin is released from cancer cells via exosomes. Apoptosis 2011; 16 (1): $1-12$.

8. Li Y, Zhang Y, Qiu F, Qiu Z. Proteomic identification of exosomal LRG1: a potential urinary biomarker for detecting NSCLC. Electrophoresis 2011; 32 (15): 1976-1983.

9. Melo SA, Luecke LB, Kahlert C et al. Glypican-1 identifies cancer exosomes and detects early pancreatic cancer. Nature 2015; 523 (7559): 177-182.

10. Skog J, Würdinger T, van Rijn S et al. Glioblastoma microvesicles transport RNA and proteins that promote tumour growth and provide diagnostic biomarkers. Nat Cell Biol 2008; 10 (12): 1470-1476.

11. Tanaka Y, Kamohara H, Kinoshita $\mathrm{K}$ et al. Clinical impact of serum exosomal microRNA-21 as a clinical biomarker in human esophageal squamous cell carcinoma. Cancer 2013; 119 (6): 1159-1167.

12. Gramantieri L, Baglioni M, Fornari $\mathbf{F}$ et al. LncRNAs as novel players in hepatocellular carcinoma recurrence. Oncotarget 2018; 9 (80): 35085-35099. 
13. Li C, Li W, Zhang Y, Zhang X, Liu T, Zhang Y, Yang Y, Wang L, Pan H, Ji J, Wang C. Increased expression of antisense IncRNA SPINT1AS1 predicts a poor prognosis in colorectal cancer and is negatively correlated with its sense transcript. OncoTargets Ther 2018; 11: 3969-3978.

14. Soekmadji C, Corcoran NM, Oleinikova I et al. Extracellular vesicles for personalized therapy decision support in advanced metastatic cancers and its potential impact for prostate cancer. Prostate. 2017; 77 (14): 1416-1423.

15. Wang YH, Ji J, Wang BC et al. Tumor-Derived Exosomal Long Noncoding RNAs as Promising Diagnostic Biomarkers for Prostate Cancer. Cell Physiol Biochem 2018; 46 (2): 532-545.

16. Gezer U, Özgür E, Cetinkaya M, Isin M, Dalay N. Long non-coding RNAs with low expression levels in cells are enriched in secreted exosomes. Cell Biol Int 2014; 38 (9): 1076-1079.

17. Yoshimoto R, Mayeda A, Yoshida M, Nakagawa S. MALAT1 long non-coding RNA in cancer. Biochim Biophys Acta 2016; 1859 (1): 192-199.

18. Taheri M, Omrani MD, Ghafouri-Fard S. Long non-coding RNA expression in bladder cancer. Biophys Rev 2017; 8: 017-0379.

19. Yuan JH, Yang F, Wang F et al. A long noncoding RNA activated by TGF- $\beta$ promotes the invasion-metastasis cascade in hepatocellular carcinoma. Cancer Cell 2014; 25 (5): 666-681.

20. Gupta RA, Shah N, Wang KC et al. Long non-coding RNA HOTAIR reprograms chromatin state to promote cancer metastasis. Nature 2010; 464: 1071-1076.

21. Prensner JR, Iyer MK, Sahu A et al. The long noncoding RNA SChLAP1 promotes aggressive prostate cancer and antagonizes the SWI/ SNF complex. Nat Genet 2013; 45: 1392-1398.

22. Hung CL, Wang LY, Yu YL et al. A long noncoding RNA connects c-Myc to tumor metabolism. Proc Natl AcadSci USA 2014; 111: 1869718702.
23. Cheng W, Zhang Z, Wang J. Long noncoding RNAs: new players in prostate cancer. Cancer Lett 2013; 339 (1): 8-14.

24. Brannan CI, Dees EC, Ingram RS, Tilghman SM. The product of the H19 gene may function as an RNA. Mol Cell Biol 1990; 10 (1): 28-36.

25. Yoshimizu T, Miroglio A, Ripoche MA et al. The H19 locus acts in vivo as a tumor suppressor. Proc Natl AcadSci USA 2008; 105 (34): $12417-12422$.

26. Yang F, Bi J, Xue X, Zheng L, Zhi K, Hua J, Fang G. Up-regulated long non-coding RNA H19 contributes to proliferation of gastric cancer cells. FEBS J 2012; 279 (17): 3159-3165.

27. Kallen AN, Zhou XB, Xu J et al. The imprinted H19 lncRNA antagonizes let-7 microRNAs. Mol Cell 2013; 52 (1): 101-112.

28. Zhu M, Chen Q, Liu X et al. lncRNA H19/miR-675 axis represses prostate cancer metastasis by targeting TGFBI. FEBS J 2014; 281 (16): 3766-3775.

29. Berteaux N, Lottin S, Adriaenssens E et al. Hormonal regulation of H19 gene expression in prostate epithelial cells. J Endocrinol 2004; 183 (1): 69-78.

30. Conigliaro A, Costa V, Lo Dico A et al. CD90+ liver cancer cells modulate endothelial cell phenotype through the release of exosomes containing H19 lncRNA. Mol Cancer 2015; 14: 155.

31. Dugimont T, Montpellier C, Adriaenssens E et al. The H19 TATAless promoter is efficiently repressed by wild-type tumor suppressor gene product p53. Oncogene 1998; 16 (18): 2395-2401.

32. Park IY, Sohn BH, Choo JH, Joe CO, Seong JK, Lee YI, Chung JH. Deregulation of DNA methyltransferases and loss of parental methylation at the insulin-like growth factor II (Igf2)/H19 loci in p53 knockout mice prior to tumor development. J Cell Biochem 2005; 94 (3): 585-596.

Received February 12, 2019. Accepted February 10, 2020. 